\title{
Appetitive Motivation Predicts the Neural Response to Facial Signals of Aggression
}

\author{
John D. Beaver, ${ }^{1}$ Andrew D. Lawrence, ${ }^{2}$ Luca Passamonti, ${ }^{1,3}$ and Andrew J. Calder ${ }^{1}$ \\ ${ }^{1}$ Medical Research Council Cognition and Brain Sciences Unit, Cambridge CB2 7EF, United Kingdom, ${ }^{2}$ Wales Institute of Cognitive Neuroscience, School of \\ Psychology, Cardiff University, Cardiff CF10 3AT, United Kingdom, and ${ }^{3}$ National Research Council, Institute of Neurological Sciences, Piano Lago di \\ Mangone, 87050 Cosenza, Italy
}

The "behavioral approach system" (BAS) (Gray, 1990) has been primarily associated with reward processing and positive affect. However, additional research has demonstrated that the BAS plays a role in aggressive behavior, heightened experience of anger, and increased attention to facial signals of aggression. Using functional magnetic resonance imaging, we show that variation in the BAS trait in healthy participants predicts activation in neural regions implicated in aggression when participants view facial signals of aggression in others. Increased BAS drive (appetitive motivation) was associated with increased amygdala activation and decreased ventral anterior cingulate and ventral striatal activation to facial signals of aggression, relative to sad and neutral expressions. In contrast, increased behavioral inhibition was associated with increased activation in the dorsal anterior cingulate, a region involved in the perception of fear and threat. Our results provide the first demonstration that appetitive motivation constitutes a significant factor governing the function of neural regions implicated in aggression, and have implications for understanding clinical disorders of aggression.

Key words: anger; facial expressions; reward; amygdala; subgenual cingulate; ventral striatum

\section{Introduction}

Aggression is a complex emotional behavior determined by both biological and environmental influences (Caspi et al., 2002; Nelson and Trainor, 2007). Elucidating the contribution of fundamental emotional systems to its expression and neural correlates is central to our understanding of aggressive disorders. Here we focus on a specific neurobiological emotional system, the "behavioral approach system” (BAS) (Gray, 1990). BAS has been primarily associated with sensitivity to reward, including goaldirected drive to pursue reward (appetitive motivation). However, individuals scoring high on this trait also show an increased tendency to display hostile and aggressive behavior (Wingrove and Bond, 1998) and heightened experience of anger (Carver, 2004). Facial signals of aggression are more likely to be interpreted as provocative by anger-prone individuals (van Honk et al., 2001; Putman et al., 2004). Hence, it is of interest that increased BAS is also associated with heightened attention to facial signals of aggression (Putman et al., 2004). The link between aggression and processing aggressive facial displays is further underlined by work demonstrating abnormal recognition

Received Sept. 7, 2007; accepted Jan. 23, 2008.

This work was supported by the United Kingdom Medical Research Council. Magnetic resonance imaging scanning was performed at the Wolfson Brain Imaging (entre (WBIC, Cambridge, UK). We thank Elisabeth von dem Hagen for assistance with the analyses and the radiographers at the WBIC. Development of the MacBrain Face Stimulus Set was overseen by Nim Tottenham and supported by the John D. and Catherine T. MacArthur Foundation Research Network on Early Experience and Brain Development. Please contact Nim Tottenham at tott0006@tc.umn.edu for more information concerning the stimulus set.

Correspondence should be addressed to Dr. Andrew J. Calder, Medical Research Council Cognition and Brain Sciences Unit, 15 Chaucer Road, Cambridge CB2 7EF, UK. E-mail: andy.calder@mrc-cbu.cam.ac.uk.

D01:10.1523/JNEUROSCI.0033-08.2008

Copyright $\odot 2008$ Society for Neuroscience $\quad$ 0270-6474/08/282719-07\$15.00/0 and neural response to angry faces in patients with aggressive disorders (Blair and Cippolotti, 2000; Best et al., 2002; Coccaro et al., 2007). How normal trait variation in BAS affects the neural correlates of aggression or aggression-related cues is currently unknown, however.

Neural models of aggression emphasize the role of structures implicated in emotion regulation, in particular the amygdala and ventromedial prefrontal cortex (vmPFC), with the former implicated in the generation of negative affect and the latter in its regulation (Davidson et al., 2000; Blair, 2003). The involvement of these structures has been identified by both human and comparative research (Davidson et al., 2000; Dielenberg and McGregor, 2001; Blair, 2003; Soloff et al., 2003; Izquierdo and Murray, 2004; Coccaro et al., 2007), with heightened aggression associated with decreased vmPFC activation (thought to reflect decreased control) and increased amygdala activation (reflecting increased negative affect) (Davidson et al., 2000; Dougherty et al., 2004).

Comparative research has also implicated the ventral striatum and its associated dopaminergic system in aggressive encounters with conspecifics (Louilot et al., 1986; Redolat et al., 1991; Vukhac et al., 2001; Ferrari et al., 2003). Similarly, human research has shown increased striatal dopamine transporter density in violent offenders (Tiihonen et al., 1995) and disrupted recognition of facial signals of aggression after damage to the ventral striatum (Calder et al., 2004). Hence, the amygdala, vmPFC, and ventral striatum were identified as regions of interest (ROIs) in our study. Using functional magnetic resonance imaging, we investigated whether the neural response of these ROIs to viewing facial signals of aggression was modulated by variation in the BAS trait in healthy participants.

The BAS is frequently contrasted with the "behavioral inhibi- 
tion system" (BIS), which is related to the expression of anxiety and the inhibition of ongoing behavior in response to threat (Gray, 1987a). Because facial signals of aggression are more likely to be interpreted as potentially dangerous by highly anxious individuals (Dimberg and Ohman, 1996), we also investigated neural modulation related to BIS.

\section{Materials and Methods}

Participants. Twenty-two right-handed healthy volunteers with normal vision and no past neurological or psychiatric history participated in the study for payment ( 13 females; mean age $=26.2$ years; $S D=7.6$ years). The National Health Service Local Research Ethics Committee for Cambridge approved the study, and all participants provided written informed consent before taking part.

Participants completed the Behavioral Inhibition/Behavioral Activation Scales (BIS/BAS) (Carver and White, 1994) after scanning. This questionnaire consists of three scales that measure traits related to behavioral approach (BAS) and one scale related to responsiveness to punishment cues (BIS). The first behavioral approach scale, BAS drive, assesses an individual's drive to engage in approach behavior in the context of potential goal or reward (i.e., appetitive motivation); questionnaire items include "When I see something I want I go all out to get it." A second BAS scale, reward responsiveness, assesses positive affect/excitability (e.g., "When good things happen to me, it affects me strongly"), and a third, fun-seeking scale, assesses the inclination to seek out new rewarding situations (e.g., "I'm always willing to try something new if I think it will be fun"). Although some research has used a BAS "total” score, structural equation modeling has shown that the three BAS measures do not form a unitary global measure and should be treated as separate scales (Ross et al., 2002). All three BAS subscales have good internal and test-retest reliability (Carver and White, 1994). We also examined the influence of the anxiety-linked trait, BIS sensitivity.

Design and procedure. Stimuli were presented in alternating $17.6 \mathrm{~s}$ blocks. Each block contained four stimuli from the same category (angry, sad, or neutral facial expressions or central fixation cross), with a total of eight blocks for each category presented in one of two pseudorandom orders. Each image was displayed for $4000 \mathrm{~ms}$ followed by a $400 \mathrm{~ms}$ interstimulus interval. Stimuli were viewed via an angled mirror above the participants' eyes, which reflected images back-projected from a translucent screen positioned in the bore of the magnet to the rear of the participants' head. For the pictures of faces, participants performed a gender discrimination task by pressing one of two keys. The facial expressions were color photographs selected from the NimStim Face Stimulus Set (Tottenham et al., 2002) (www.macbrain.org). There were 16 exemplars (half female) for each face type.

Imaging and statistical analysis. Magnetic resonance imaging scanning was performed on a $3 \mathrm{~T}$ Bruker Medspec scanner (Bruker, Ettlingen, Germany) with a head-coil gradient set. Whole-brain data were acquired with echo-planar T2*-weighted (EPI) imaging, sensitive to blood oxygenation level-dependent (BOLD) signal contrast (21 interleaved 4-mmthick slices; $1 \mathrm{~mm}$ interslice gap; repetition time $=1100 \mathrm{~ms}$; echo time $=$ $30 \mathrm{~ms}$; $65^{\circ}$ flip angle; $24 \times 24 \mathrm{~cm}$ field of view; $64 \times 64$ matrix; $144 \mathrm{kHz}$ voxel bandwidth). Slice acquisition was transverse oblique, angled to avoid the eyeballs. The first 15 volumes were dummy scans and were discarded to allow for equilibration effects.

Data were analyzed using SPM2 software (Wellcome Trust Centre for Neuroimaging, London, UK). EPI images were corrected for slice timing and head movement, and phase-map-derived undistortion was applied (Cusack et al., 2003). The mean functional image was calculated for each participant and inspected for excessive signal dropout in medial temporal and ventral prefrontal cortices. EPI and structural images were coregistered, normalized to a standard template, and smoothed with an $8 \mathrm{~mm}$ full-width at half-maximum Gaussian kernel. Condition effects were estimated using boxcar regressors convolved with a canonical hemodynamic response function in a general linear model. Low-frequency signal drift was removed with a high-pass filter (cutoff, $128 \mathrm{~s}$ ).

A random-effects model was implemented using a two-stage process, of within- and between-subjects modeling in turn. This random-effects analysis assessed effects on the basis of intersubject variance and thus allowed inferences about the population that the participants were drawn from. For each participant, we used a general linear model to assess regionally specific effects of task parameters on BOLD indices of activation. The model included three experimental factors (angry, sad, and neutral facial expressions) and effects of no interest (realignment parameters) to account for motion-related variance. Individual first-level contrast images were generated for the contrasts of angry versus neutral, angry versus sad, and sad versus neutral faces.

The second level models included each of the 22 contrast images from the participants' first-level analysis, subjected to a voxelwise contrast and $t$ test using Gaussian random field theory to assess statistical significance (Friston et al., 1995). A one-sample $t$ test was first used to identify the brain regions activated for any specific contrast. Then, simple regression models were performed to detect regions where BOLD contrast estimates correlated with subscales of the BIS/BAS questionnaire. We defined vmPFC cortex, amygdala, and ventral striatum as ROIs, because these areas have previously been implicated in aggression. To sample activity within these regions, we used 10 - $\mathrm{mm}$-radius sphere centered on \pm 18 , $-6,-18$ for the amygdala, a 12-mm-radius sphere centered on $\pm 9,33$, -12 for the ventromedial prefrontal cortex, and a 10-mm-radius sphere centered $\pm 9,15,-3$ for the ventral striatum [coordinates from Bray and O'Doherty (2007)]. Summary statistical maps were thresholded at $p<$ 0.005 uncorrected, and for a priori ROIs, we used small-volume correction for multiple comparisons applied at $p<0.05$, as a more stringent test of our a priori hypotheses (Worsley et al., 1996). All regions surviving $p<$ 0.001 uncorrected for 10 contiguous voxels are also reported in Tables 1 and 2. Supplemental Table 1 summarizes group-level contrasts, regardless of individual difference in BAS. Localization of activations was checked with reference to the atlases of Duvernoy (1999) and Damasio (1995). For visualizing activations, group maps are overlaid on a representative high-resolution structural T1-weighted image from the SPM2 canonical image set, coregistered to Montreal Neurological Institute (MNI) space. Activations are reported using $(x, y, z)$ coordinates in MNI standardized space.

\section{Results}

We were primarily interested in the BAS-drive subscale measuring goal/incentive-oriented appetitive motivation, because it has been most consistently associated with aggression-related measures, including attention to facial signals of aggression (Harmon-Jones, 2003; Putman et al., 2004; Smits and Kuppens, 2005). Analyses also examined any relationship with the other two BAS scales (reward responsive and fun seeking) and the BIS scale. The range, mean, and SD of our sample on each scale were as follows: BAS drive, 7-15; mean $=10.40$; $\mathrm{SD}=1.97$; reward responsiveness, $15-20$; mean $=16.86 ; \mathrm{SD}=1.64$; fun seeking, $7-15 ;$ mean $=12.46$; $\mathrm{SD}=2.19 ; \mathrm{BIS}, 16-27$; mean $=21.09 ; \mathrm{SD}=$ 2.62). BAS subscale scores were not significantly correlated with BIS scores ( $r$ values $<0.16$; $p$ values $>0.5$ ). These scores are similar to previously published norms (Carver and White, 1994).

The relationship between individual differences in BAS-drive scores and change in BOLD response to angry and sad faces for the three a priori ROIs [amygdala, vmPFC cortex, and ventral striatum (see Materials and Methods)] was determined with separate simple regressions between BAS drive and each of three contrasts [angry vs neutral, angry vs sad, and sad vs neutral (Table $1)$ ]; additional regions surpassing $p<0.001$ with 10 contiguous voxels are also reported. Significant brain regions across all participants (regardless of BAS scores) were also estimated for the same and additional contrasts (supplemental Table 1, available at www.jneurosci.org as supplemental material).

In line with our predictions, BAS-drive scores were positively correlated with change in BOLD signal in the left amygdala for both the angry versus neutral [MNI coordinates $(x y z),-18,0$, $-24 ; t=4.82 ; p<0.005$ small-volume corrected (SVC); $r=0.73$; 
Table 1. Regions showing positive and negative correlations between BAS-drive scores and activation to angry and sad facial expressions

\begin{tabular}{|c|c|c|c|c|c|c|c|c|}
\hline \multirow[b]{2}{*}{ Contrast } & \multirow[b]{2}{*}{ Cerebral region } & \multirow[b]{2}{*}{ Side } & \multirow[b]{2}{*}{ No. of voxels } & \multirow[b]{2}{*}{$p$ value } & \multirow[b]{2}{*}{$t$ value } & \multicolumn{3}{|c|}{ MNI coordinates } \\
\hline & & & & & & $x$ & $y$ & $z$ \\
\hline \multicolumn{9}{|c|}{ Angry -neutral } \\
\hline Positive & Amygdala*** & $\mathrm{L}$ & 20 & 0.0001 & 4.82 & -18 & 0 & -24 \\
\hline \multirow[t]{7}{*}{ Negative } & Ventral anterior cingulate cortex* & $\mathrm{L}$ & 90 & 0.0001 & 3.99 & -15 & 36 & -12 \\
\hline & Ventral anterior cingulate cortex** & $\mathrm{R}$ & 233 & 0.0001 & 4.65 & 9 & 39 & -9 \\
\hline & Ventral striatum* & $\mathrm{R}$ & & 0.0001 & 4.43 & 18 & 18 & -6 \\
\hline & Dorsal striatum & $\mathrm{R}$ & & 0.0001 & 4.29 & 21 & 15 & 9 \\
\hline & Inferior parietal lobule & $\mathrm{L}$ & 81 & 0.0001 & 4.93 & -39 & -72 & 39 \\
\hline & Retrosplenial cortex & $\mathrm{L}$ & 203 & 0.0001 & 4.32 & -3 & -42 & 15 \\
\hline & Dorsolateral prefrontal cortex & $\mathrm{R}$ & 52 & 0.0001 & 4.14 & 39 & 9 & 42 \\
\hline \multicolumn{9}{|l|}{ Angry-sad } \\
\hline Positive & Amygdala** & $\mathrm{L}$ & 21 & 0.0001 & 4.41 & -18 & 3 & -24 \\
\hline \multirow[t]{5}{*}{ Negative } & Inferior parietal lobule & $\mathrm{L}$ & 38 & 0.0001 & 5.04 & -42 & -63 & 12 \\
\hline & Retrosplenial cortex & $\mathrm{R}$ & 141 & 0.0001 & 4.34 & 12 & -39 & 36 \\
\hline & Ventral anterior cingulate cortex* & $\mathrm{L}$ & 5 & 0.0001 & 3.90 & -18 & 39 & -12 \\
\hline & Ventral anterior cingulate cortex & $\mathrm{R}$ & 8 & 0.001 & 3.55 & 18 & 39 & -12 \\
\hline & Ventral striatum $^{b}$ & $\mathrm{R}$ & 17 & 0.002 & 3.21 & 6 & 24 & -6 \\
\hline \multicolumn{9}{|l|}{ Sad-neutral } \\
\hline \multirow[t]{2}{*}{ Negative } & Dorsal striatum & $\mathrm{L}$ & 208 & 0.001 & 3.71 & -27 & -18 & 6 \\
\hline & & $\mathrm{R}$ & 187 & 0.0001 & 4.15 & 30 & -12 & 9 \\
\hline
\end{tabular}

A priori regions of interest (shown in bold) are small-volume corrected [ ${ }^{*} p<0.05$ SVC; ${ }^{* *} p<0.02$ SVC; ${ }^{* * *} p<0.005$ SVC; $0.1>p>0.05$ SVC (borderline); see Materials and Methods for ROls]. All other activations surpassing a threshold of $p<0.001$ uncorrected, minimum 10 contiguous voxels, are also shown. Coordinates are in MNI standardized space. L, Left; $R$, right.

Table 2. Regions showing positive and negative correlations between BIS scores and activation to angry and sad facial expressions

\begin{tabular}{|c|c|c|c|c|c|c|c|c|}
\hline \multirow[b]{2}{*}{ Contrast } & \multirow[b]{2}{*}{ Cerebral region } & \multirow[b]{2}{*}{ Side } & \multirow[b]{2}{*}{ No. of voxels } & \multirow[b]{2}{*}{$p$ value } & \multirow[b]{2}{*}{$t$ value } & \multicolumn{3}{|c|}{ MNI coordinates } \\
\hline & & & & & & $x$ & $y$ & $z$ \\
\hline \multicolumn{9}{|c|}{ Angry-neutral } \\
\hline Positive & Dorsal anterior cingulate cortex & L & 53 & 0.0001 & 4.15 & -6 & 33 & 24 \\
\hline Negative & Nothing at $p<0.005$, uncorrected & & & & & & & \\
\hline \multicolumn{9}{|l|}{ Angry-sad } \\
\hline Positive & Dorsolateral prefrontal cortex & $\mathrm{R}$ & 31 & 0.001 & 3.71 & 57 & 15 & 27 \\
\hline Negative & Posterior cingulate cortex & $\mathrm{L}$ & 77 & 0.001 & 3.60 & -12 & -33 & 42 \\
\hline
\end{tabular}

$p<0.001$ uncorrected, minimum 10 contiguous voxels. Coordinates are in MNI standardized space. L, Left; $R$, right.

$p<0.001]$ and angry versus sad contrasts $(-18,3,-24 ; t=4.41$; $p<0.02$ SVC; $r=0.70 ; p<0.001)$ but not for the sad versus neutral contrast. Thus, the amygdala responses to the sight of angry faces increased as a function of increased BAS drive (Fig. 1). In contrast, a negative relationship was found between BAS-drive scores and BOLD signal in the ventral anterior cingulate cortex for both angry versus neutral (left, $-15,36,-12 ; t=3.99 ; p<$ 0.05 SVC; $r=-0.66 ; p<0.001$; right, $9,39,-9 ; t=4.65 ; p<$ 0.02 SVC; $r=-0.72 ; p<0.001)$ and angry versus sad (left, -18 , $39,-12 ; t=3.90 ; p<0.05$ SVC; $r=-0.65 ; p<0.001 ;$ right, 18 , $39,-12 ; t=3.55 ; p=0.085$ SVC; $r=-0.62 ; p<0.001$ ) (Fig. 2) contrasts, with decreased activity for these contrasts associated with increased BAS drive. A similar negative correlation with BAS drive was also found in the ventral striatum for angry versus neutral $(18,18,-6 ; t=4.43 ; p<0.05$ SVC; $r=-0.70 ; p<0.001)$ and a borderline effect in a slightly anterior position, for angry versus sad contrasts $(6,24,-6 ; t=3.21 ; p=0.097$ SVC; $r=$ $-0.58 ; p<0.001$ ) (Fig. 3), although the latter may form part of the more anterior ventral anterior cingulate activation. No correlations with BAS-drive scores were found for the sad versus neutral contrast in the a priori ROIs (Table 1). The angry versus neutral contrast also produced a negative correlation with BAS drive in the right dorsolateral prefrontal cortex $(39,9,42 ; t=$ $4.14 ; p<0.0001$ uncorrected; $r=-0.67 ; p<0.001$ ), a region also implicated in emotion regulation (Davidson et al., 2000). Supplemental Figures 1 and 2 (available at www.jneurosci.org as supplemental material) show that the correlations in the amygdala, ventral anterior cingulate, and ventral striatum for the angry versus neutral and angry versus sad contrasts persist when the participant with the highest BAS-drive score was excluded. Hence, a single outlier did not drive these correlations.

The consistent correlations with BAS drive in the amygdala, ventral anterior cingulate cortex, and ventral striatum across both the angry versus neutral and angry versus sad contrasts, and absence for the sad versus neutral comparison, indicate that the relationship with BAS drive was restricted to the anger contrasts, rather than a generalized modulation of all emotional stimuli.

The principal aim of the present study was to examine whether individual differences in BAS drive modulate the neural response to signals of aggression in others. For completeness, however, we also explored potential relationships with BASreward responsiveness and BAS-fun seeking in the a priori ROIs. Fun seeking showed a borderline negative correlation for the angry versus sad contrast in the left and right hippocampi (left, $-27,-9,-21 ; t=3.48 ; p<0.001$, uncorrected; $r=-0.66 ; p<$ 0.001 ; right, $15,-12,-15 ; t=3.60,<0.001$, uncorrected; $r=$ $-0.63 ; p<0.005)$; no other effects approached significance. Thus, our results are consistent with behavioral studies that most consistently highlight an association between the BAS-drive scale of the BIS/BAS questionnaires and measures of aggression or attention to facial signals of aggression (Harmon-Jones, 2003; Putman et al., 2004; Smits and Kuppens, 2005).

Finally, we examined the data with respect to participants' BIS scores. We found that BIS scores were positively correlated with BOLD signal changes in the left dorsal anterior cingulate cortex for the angry versus neutral contrast, and left dorsolateral pre- 

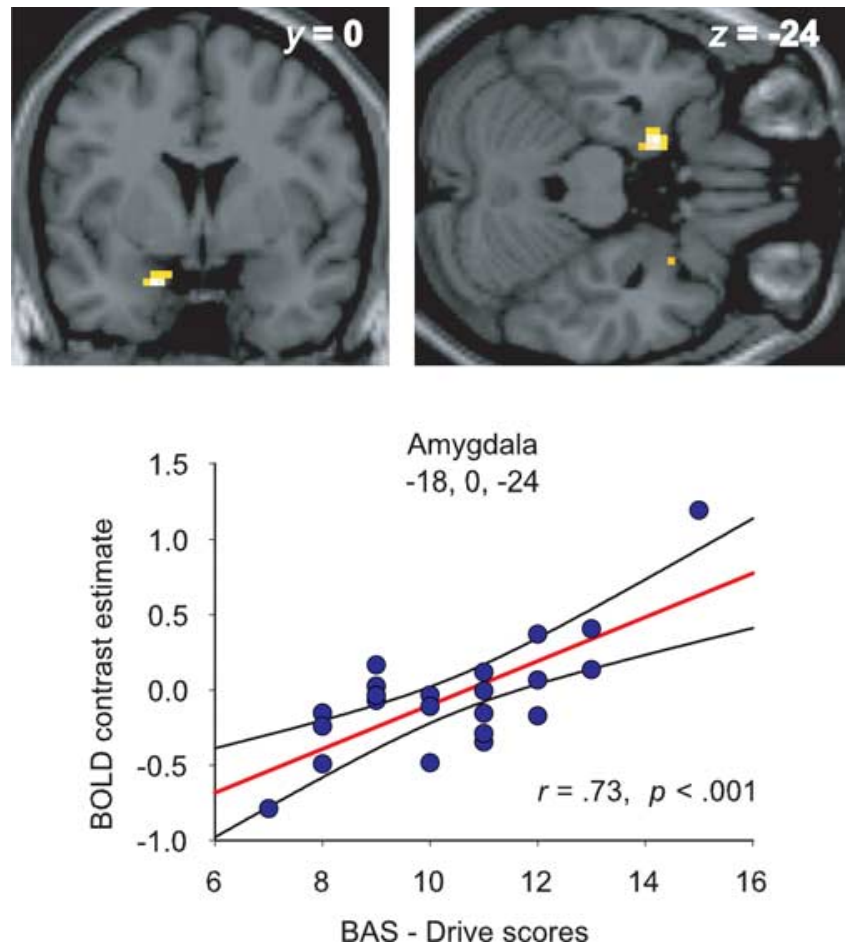

Figure 1. Significant modulation of the amygdala response to facial signals of aggression (relative to neutral expressions) by BAS drive. The scatter plot shows the BOLD signal change in the peak-activated voxel for this contrast, plotted as a function of participants' $B$ AS-drive scores. The regression line and $95 \%$ confidence intervals are shown. Activations are thresholded at $p<$ 0.001 , uncorrected.

frontal cortex for angry versus sad, the reverse correlation of that observed for BAS (Table 2). In addition, the posterior cingulate cortex also showed a negative correlation for the angry versus sad contrast.

\section{Discussion}

Our study demonstrates for the first time that the response of brain regions implicated in aggression is modulated as a function of appetitive motivation (BAS drive) when participants view facial signals of aggression relative to both sad and neutral faces. With increasing BAS drive, amygdala activity increased, whereas activity in the ventral anterior cingulate and ventral striatum decreased. In contrast, individual variation in BIS, which underlies anxiety, produced a positive correlation in the dorsal anterior cingulate, a region implicated in fear conditioning (Phelps et al., 2004), anticipation of aversive events (Nitschke et al., 2006), and "urgent" response inhibition (Garavan et al., 2002). These different effects of BIS and BAS accord with the proposal that a threatening stimulus can be interpreted as provocative (inducing aggression) or dangerous (inducing anxiety), depending on an individual's temperament or environmental context (Beck, 1976; Dimberg and Ohman, 1996; van Honk et al., 2001).

\section{BAS, aggression and reward}

BAS has been implicated in both reward-based and aggressive behavior (Carver and White, 1994; Carver, 2004). Although not immediately apparent, a link between reward and aggression is evident at multiple levels. First, both are associated with approach behavior (Carver, 2004). Second, an adaptive function of aggression relates to the forced acquisition and protection of valued resources, such as food, reproductive partners, territory, and so-
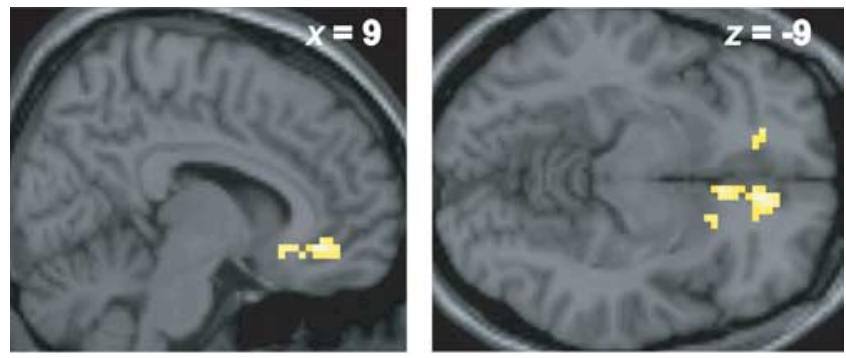

Ventral anterior cingulate cortex

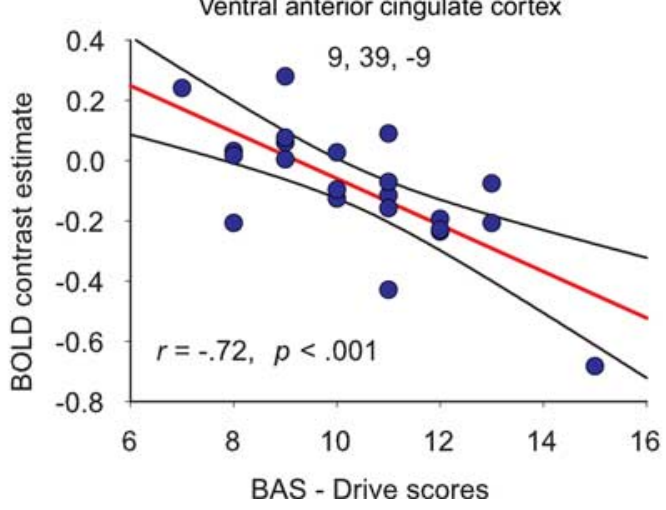

Figure 2. Significant modulation of the ventral anterior cingulate response to facial signals of aggression (relative to neutral expressions) by BAS drive. The scatter plot shows the BOLD signal change in peak-activated voxel for this contrast, plotted as a function of participants' BAS-drive scores. The regression line and $95 \%$ confidence intervals are shown. Activations are thresholded at $p<0.001$, uncorrected.

cial status (Blanchard and Blanchard, 2003); aggression is also instigated by the omission or termination of rewards (Rolls, 1990; Berkowitz and Harmon-Jones, 2004). Third, the structures we identified (ventral striatum, amygdala, and ventral anterior cingulate) have been implicated in both aggression and reward processing (Louilot et al., 1986; Davidson et al., 2000; Berridge and Robinson, 2003), and each presents a dense dopaminergic innervation, a neurochemical primarily associated with reward, but also aggression (Redolat et al., 1991; Tiihonen et al., 1995; Ferrari et al., 2003; Nelson and Trainor, 2007), the recognition of facial signals of aggression (Lawrence et al., 2002), and the behavioral approach system in general (Gray, 1987b; Reuter et al., 2006). Note also that the aggression seen in psychopathy [a condition associated with high BAS activity (Cole and Zahn-Waxler, 1992; Gray, 1994)] is frequently goal directed, linked to the gain of monetary and sexual rewards or social status (Cornell et al., 1996).

The reduced frontostriatal response with increasing BAS drive was associated with increased amygdala activity, a similar pattern to the experience of aggression (Dougherty et al., 2004). Similarly, individuals with intermittent explosive disorder show an exaggerated amygdala response and reduced vmPFC response to aggressive facial expressions relative to controls (Coccaro et al., 2007), whereas a comparable exaggerated pattern is found in participants carrying the low-expression variant of the MAO-A (monoamine oxidize A) polymorphism (Meyer-Lindenberg et al., 2006), associated with increased risk for violent behavior (Caspi et al., 2002). However, whereas these previous effects were observed relative to low-level baselines, our use of neutral/sad baselines shows that our current effects do not reflect a more general response to faces or facial expressions.

Although previous research shows that damage to the amygdala, ventral striatum, and ventral prefrontal cortex can impair 


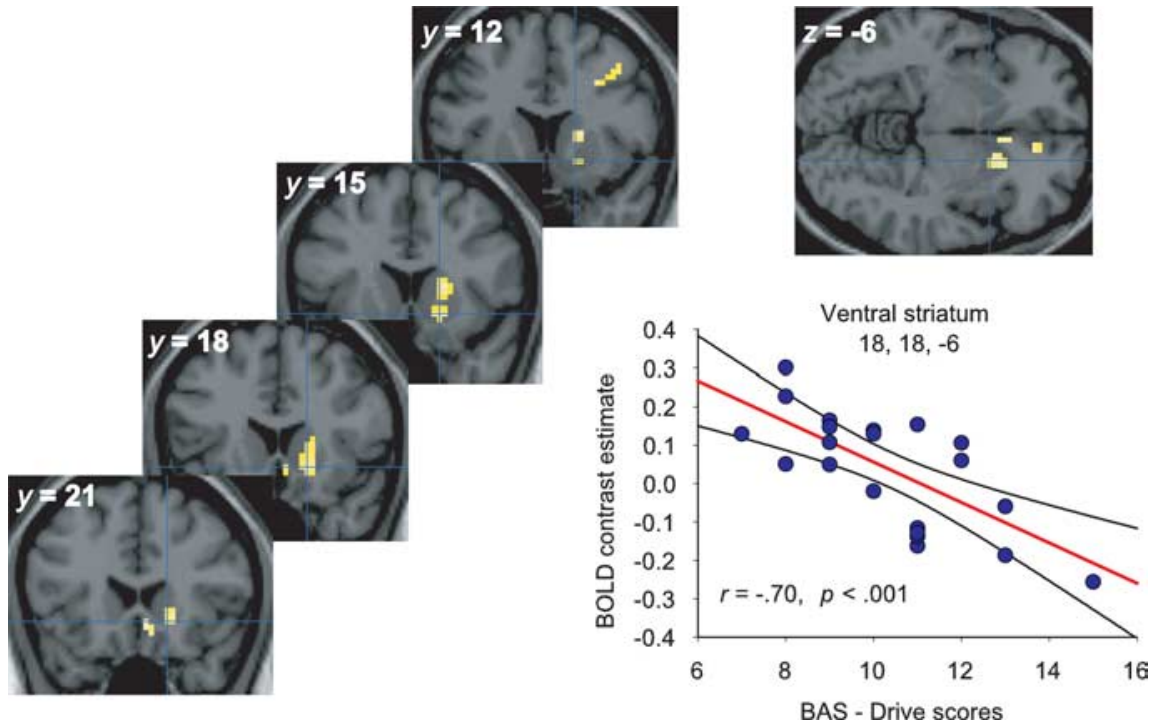

Figure 3. Significant modulation of the ventral striatal response to facial signals of aggression (relative to neutral expressions) by BAS drive. The scatter plot shows the BOLD signal change in peak-activated voxel for this contrast, plotted as a function of participants' BAS-drive scores. The regression line and $95 \%$ confidence intervals are shown. Activations are thresholded at $p<$ 0.001 , uncorrected.

recognition of facial expressions of anger (Calder et al., 1996; Blair and Cippolotti, 2000; Sato et al., 2002; Calder et al., 2004), we are not claiming that these areas are specific to aggression or signals of anger. The amygdala and vmPFC/anterior cingulate in particular have an established role in emotion regulation (Davidson et al., 2000). For example, an inverse relationship between these regions is found during the suppression/regulation of negative affect in response to emotional scenes (Urry et al., 2006) (see also van Reekum et al., 2007). The amygdala and ventromedial prefrontal cortex also respond to ambiguous facial expressions, with decreased vmPFC and increased amygdala activation associated with a negative interpretation, and the opposite with a positive interpretation (Kim et al., 2003); again, Kim et al., (2003) attribute this to differences in the modulatory influence of the vmPFC on the amygdala. An inverse relationship is also observed during fear conditioning (Milad et al., 2007). Hence, we propose that the effects we have observed reflect the interactive effects of BAS and aggressive facial signals (Harmon-Jones, 2003; Putman et al., 2004) on this emotion regulation system, and not a neural system that is specific to aggression per se.

Comparative research has highlighted the ventral striatum's role in aggression (Louilot et al., 1986; Redolat et al., 1991; Ferrari et al., 2003). Hence, it is of interest that this region showed the same negative relationship with BAS drive found in the ventral anterior cingulate. Both regions are interconnected (Ferry et al., 2000) and are involved in the control and suppression of emotional behavior. Damage to the vmPFC and ventral striatum in humans and animals increases impulsive and aggressive behavior (Grafman et al., 1996; Anderson et al., 1999; Cardinal et al., 2001; Izquierdo and Murray, 2004). In addition, neuroimaging research has shown abnormal frontostriatal activation in aggressive individuals with borderline personality disorder, reflecting reduced serotonergic function (Leyton et al., 2001), a neurotransmitter implicated in aggression (Nelson and Trainor, 2007). Thus, the blunted frontal-striatal activation with increasing BAS drive might represent a reduced ability to suppress impulsive negative reactions to signals of conspecific challenge.

It is of interest that the blocking of goal-directed behavior by external agents is a major instigator of aggression (Berkowitz and Harmon-Jones, 2004). In our own study, "goal-directed" behavior could be conceived as each individual's trait tendency to engage in this behavior (i.e., BAS drive), and the aggressive faces as blocks/obstructions. In other words, the more goal-oriented/motivated an individual is, the more likely is conspecific challenge (or any block/obstruction) to make them angry, and therefore engage the neural correlates of aggression.

\section{BIS and dorsal anterior cingulate}

In contrast to the blunted ventral anterior cingulate response to angry expressions found in high-BAS individuals, increasing BIS produced the opposite positive correlation in the dorsal anterior cingulate. In Gray's original hypothesis (Gray, 1982), the behavioral inhibition system is engaged by novel and threatening stimuli, causing inhibition of ongoing behavior and anxiety. Initially, the septohippocampal system was considered the main neural substrate of BIS (Gray, 1982), but a more recent formulation (McNaughton and Corr, 2004) has highlighted the contribution of the $\mathrm{mPFC} /$ anterior cingulate. Comparative research has identified a distinction between the ventral mPFC, associated with an inhibitory role in fear expression, and a more dorsal $\mathrm{mPFC}$ region associated with an excitatory role (Quirk and Beer, 2006). Similarly, human research has emphasized the role of the vmPFC regions in regulation/suppression of emotion (Davidson et al., 2000; Dougherty et al., 2004; Coccaro et al., 2007), and dorsal $\mathrm{mPFC} /$ anterior cingulate in fear conditioning (Buchel et al., 1998; Phelps et al., 2004), "urgent" response inhibition (Garavan et al., 2002), and anticipation of aversive events (a central component of anxiety) (Nitschke et al., 2006). Thus, the dorsal anterior cingulate correlation with BIS may reflect an increased susceptibility to an anxiety or fear response to threatening stimuli (i.e., angry faces). The correlation with BIS was evident for the angry versus neutral contrast but not angry versus sad. Hence, although the modulation of the amygdala and ventral anterior cingulate response by BAS was evident relative to both neutral and sad baselines, the effect for BIS was less specific, but notably did not generalize to sad versus neutral faces.

Two further points should be emphasized. First, the effects we have reported are in healthy volunteers with no known psychiatric history. Hence, they reflect the divergent modulatory influences of distinct emotional traits, linked to increased risk for aggressive (BAS) or anxious (BIS) behavior, on healthy brain systems. Second, inspection of supplemental Table 1 (available at www.jneurosci.org as supplemental material), summarizing group-based analyses regardless of individual differences, shows that the full extent of the inverse relationship between frontostriatal and amygdala regions to aggressive faces is only clearly evident when taking individual differences in BAS drive into account. This accords with previous research showing only variable evidence of an amygdala response to angry expressions in groupbased analyses (Whalen et al., 2001; Murphy et al., 2003; Strauss et al., 2005). Similarly, the dorsal anterior cingulate was only found when individual variation in BIS was incorporated into the analysis. In other words, the neural circuitry underlying the pro- 
cessing of angry expressions is only fully realized by taking variation in relevant emotional dimensions into consideration. This accords with other recent research emphasizing the importance of individual differences to neural responses to food stimuli (Beaver et al., 2006; Calder et al., 2007) or happy and fearful facial expressions (Canli et al., 2002; Bishop et al., 2004).

We have shown that the interaction between BAS drive and the perception of facial signals of aggression operates directly on regions implicated in aggressive disorders, demonstrating the potential importance of trait variation in BAS to understanding the psychological and neural bases of these conditions. In contrast, but in accord with its posited neural substrates, variation in BIS identified a region implicated in anxiety and fear. Although research to date has focused on the relationship between BAS and reward-based processing, understanding its relationship to aggression provides a wider understanding of this personality trait's role in emotion and emotional systems underlying individual differences in aggression.

\section{References}

Anderson SW, Bechara A, Damasio H, Tranel D, Damasio AR (1999) Impairment of social and moral behavior related to early damage in human prefrontal cortex. Nat Neurosci 2:1032-1037.

Beaver JB, Lawrence AD, van Ditzhuijzen J, Davis MH, Woods A, Calder AJ (2006) Individual differences in reward drive predict neural responses to images of food. J Neurosci 26:5160-5166.

Beck (1976) Cognitive therapy and emotional disorders. New York: International Universities.

Berkowitz L, Harmon-Jones E (2004) Toward an understanding of the determinants of anger. Emotion 4:107-130.

Berridge KC, Robinson TE (2003) Parsing reward. Trends Neurosci 26:507-513.

Best M, Williams JM, Coccaro E (2002) Evidence for a dysfunctional prefrontal circuit in patients with an impulsive aggressive disorder. Proc Natl Acad Sci USA 99:8448-8453.

Bishop SJ, Duncan J, Brett M, Lawrence AD (2004) Prefrontal cortical function and anxiety: controlling attention to threat-related stimuli. Nat Neurosci 7:184-188.

Blair RJR (2003) The neurobiological basis of psychopathy. Br J Psychiatry 182:5-7.

Blair RJR, Cippolotti L (2000) Impaired social response reversal: a case of "acquired sociopathy." Brain 123:1122-1141.

Blanchard RJ, Blanchard DC (2003) What can animal aggression research tell us about human aggression? Horm Behav 44:171-177.

Bray S, O’Doherty J (2007) Neural coding of reward-prediction error signals during classical conditioning with attractive faces. J Neurophysiol 97:3036-3045.

Buchel C, Morris J, Dolan RJ, Friston KJ (1998) Brain systems mediating aversive conditioning: an event-related fMRI study. Neuron 20:947-957.

Calder AJ, Young AW, Rowland D, Perrett DI, Hodges JR, Etcoff NL (1996) Facial emotion recognition after bilateral amygdala damage: differentially severe impairment of fear. Cogn Neuropsychol 13:699-745.

Calder AJ, Keane J, Lawrence AD, Manes F (2004) Impaired recognition of anger following damage to the ventral striatum. Brain 127:1958-1969.

Calder AJ, Beaver JD, Davis MH, van Ditzhuijzen J, Keane J, Lawrence AD (2007) Disgust sensitivity predicts the insula and pallidal response to pictures of disgusting foods. Eur J Neurosci 425:3422-3428.

Canli T, Sivers H, Whitfiled SL, Gotlib IH, Gabrieli JDE (2002) Amygdala response to happy faces as a function of extraversion. Science 295:2191.

Cardinal RN, Pennicott DR, Sugathapala CL, Robbins TW, Everitt BJ (2001) Impulsive choice induced in rats by lesions of the nucleus accumbens core. Science 292:2499-2501.

Carver CS (2004) Negative affects deriving from the behavioral approach system. Emotion 4:3-22.

Carver CS, White TL (1994) Behavioral inhibition, behavioral activation, and affective responses to impending reward and punishment- - the BIS BAS scales. J Pers Soc Psychol 67:319-333.

Caspi A, McClay J, Moffit TE, Mill J, Martin J, Craig IW, Taylor A, Poulton R (2002) Role of genotype in the cycle of violence in maltreated children. Science 297:851-854.
Coccaro EF, McCloskey MS, Fitzgerald DA, Phan KL (2007) Amygdala and orbitofrontal reactivity to social threat in individuals with impulsive aggression. Biol Psychiatry 62:168-178.

Cole PM, Zahn-Waxler C (1992) Emotional dysregulation in disruptive behavior disorders. In: Rochester symposium on developmental psychopathology, Vol 4, Developmental perspectives on depression (Ciccheti D, Toth SL, eds), pp 173-210. Rochester, NY: University of Rochester.

Cornell DG, Warren J, Hawk G, Stafford E, Oram G, Pine D (1996) Psychopathy in instrumental and reactive violent offenders. J Consult Clin Psychol 64:783-790.

Cusack R, Brett M, Osswald K (2003) An evaluation of the use of magnetic field maps to undistort echo-planar images. NeuroImage 18:127-142.

Damasio H (1995) Human brain anatomy in computerized images. New York: Oxford UP.

Davidson RJ, Putnam KM, Larson CL (2000) Dysfunction in the neural circuitry of emotion regulation - a possible prelude to violence. Science 289:591-594.

Dielenberg RA, McGregor IS (2001) Defensive behavior in rats towards predatory odors: a review. Neurosci Biobehav Rev 25:597-609.

Dimberg U, Ohman A (1996) Behold the face of wrath: psychophysiological responses to facial stimuli. Motiv Emot 20:149-182.

Dougherty DD, Rauch SL, Deckersbach T, Marci C, Loh R, Shin LM, Alpert NM, Fischman AJ, Fava M (2004) Ventromedial prefrontal cortex and amygdala dysfunction during anger induction positron emission tomography in patients with major depressive disorder with anger attacks. Arch Gen Psychiatry 61:795-804.

Duvernoy HM (1999) The human brain. Vienna: Springer.

Ferrari PF, van Erp AMM, Rornatzky W, Miczek KA (2003) Accumbal dopamine and serotonin in anticipation of the next aggressive episode in rats. Eur J Neurosci 17:371-378.

Ferry AT, Ongur D, Xinhai AN, Price JL (2000) Prefrontal cortical projections to the striatum in macaque monkeys: evidence for an organization related prefrontal network. J Comp Neurol 425:447-470.

Friston KJ, Holmes AP, Worsley KJ, Poline J-B, Frith CD, Frackowiak RSJ (1995) Statistical parametric maps in functional imaging: a general linear approach. Hum Brain Mapp 2:189-210.

Garavan H, Ross TJ, Murphy K, Roche RAP, Stein EA (2002) Dissociable executive functions in the dynamic control of behavior: inhibition, error detection, and correction NeuroImage 17:1820-1829.

Grafman J, Schwab K, Warden D, Pridgen A, Brown HR, Salazar AM (1996) Frontal lobe injuries, violence, and aggression: a report of the Vietnam Head Injury Study. Neurology 46:1231-1238.

Gray JA (1982) The neuropsychology of anxiety: an enquiry into the functions of the septo-hippocampal system, Ed 1. Oxford: Oxford UP.

Gray JA (1987a) The psychology of fear and stress, Ed 2. Cambridge: Cambridge UP.

Gray JA (1987b) Perspectives on anxiety and impulsivity: a commentary. J Res Pers 21:493-509.

Gray JA (1990) Brain systems that mediate both emotion and cognition. Cogn Emot 4:269-288

Gray JA (1994) Framework for taxonomy for psychiatric disorders. In: Emotions: essays in emotion theory (van Goozen SHM, van de Pol NE, Sergent JA, eds), pp 29-59. Hillsdale, NJ: Lawrence Erlbaum.

Harmon-Jones E (2003) Anger and the behavioural approach system. Pers Individ Dif 35:995-1005.

Izquierdo A, Murray EA (2004) Combined unilateral lesions of the amygdala and orbital prefrontal cortex impair affective processing in rhesus monkeys. J Neurophysiol 91:2023-2039.

Kim H, Somerville LH, Johnstone T, Alexander AL, Whalen PJ (2003) Inverse amygdala and medial prefrontal cortex responses to surprised faces. NeuroReport 14:2317-2322.

Lawrence AD, Calder AJ, McGowan SW, Grasby PM (2002) Selective disruption of the recognition of facial expressions of anger. NeuroReport 13:881-884.

Leyton M, Okazawa H, Diksic M, Paris J, Rosa P, Mzengeza S, Young SN, Blier P, Benkelfat C (2001) Brain regional $\alpha$ - $\left[{ }^{11} \mathrm{C}\right]$ methyl-L-tryptophan trapping in impulsive subjects with borderline personality disorder. Am J Psychiatry 158:775-782.

Louilot A, LeMoal M, Simon H (1986) Differential reactivity of dopaminergic neurons in the nucleus accumbens in response to different behavioral situations. An in vivo voltammetric study in freely moving rats. Brain Res 395-400. 
McNaughton N, Corr PJ (2004) A two-dimensional neuropsychology of defense: fear/anxiety and defensive distance. Neurosci Behav Rev 28:285-305.

Meyer-Lindenberg A, Buckholtz JW, Kolachana B, Hariri A, Pezawas L, Blasi G, Wabnitz A, Honea R, Verchinsk B, Callicott J, Egan M, Mattay V, Weinberger DR (2006) Neural mechanisms of genetic risk for impulsivity and violence in humans. Proc Natl Acad Sci USA 103:6269-6274.

Milad MR, Wright CI, Orr SP, Pitman RK, Quirk GJ, Rauch SL (2007) Recall of fear extinction in humans activates the ventromedial prefrontal cortex and hippocampus in concert. Biol Psychiatry 62:446-454.

Murphy FC, Nimmo-Smith I, Lawrence AD (2003) Functional neuroanatomy of emotion: a meta-analysis. Cogn Affect Behav Neurosci 3:207-233.

Nelson RJ, Trainor BC (2007) Neural mechanisms of aggression. Nat Rev Neurosci 8:536-546.

Nitschke JB, Sarinopoulos I, Mackiewicz K, Schaefer HS, Davidson RJ (2006) Functional neuroanatomy of aversion and its anticipation. NeuroImage 29:106-116.

Phelps EA, Delgado MR, Nearing KI, LeDoux JE (2004) Extinction learning in humans: role of the amygdala and vmPFC. Neuron 43:897-905.

Putman P, Hermans E, van Honk J (2004) Emotional Stroop performance for masked angry faces: it's BAS, not BIS. Emotion 4:305-311.

Quirk GJ, Beer JS (2006) Prefrontal involvement in the regulation of emotion: convergence of rat and human studies. Curr Opin Neurobiol 16:723-727.

Redolat R, Brain PF, Simón VM (1991) Sulpiride has an antiaggressive effect in mice without markedly depressing motor activity. Neuropsychopharmacology 30:41-46.

Reuter M, Schmitz A, Corr P, Hennig J (2006) Molecular genetics support Gray's personality theory: the interaction of COMT and DRD2 polymorphisms predicts the behavioural approach system. Int J Neuropsychopharmacol 9:155-166.

Rolls ET (1990) A theory of emotion, and its application to understanding the neural basis of emotion. Cogn Emot 4:161-190.

Ross SR, Millis SR, Bonebright TL, Bailley SE (2002) Confirmatory factor analysis of the behavioral inhibition and activation scales. Pers Individ Dif 33:861-865.

Sato W, Kutota Y, Okada T, Murai T, Yoshikawa S, Sengoku A (2002) Seeing happy emotion in fearful and angry faces: qualitative analysis of facial expression recognition in a bilateral amygdala-damaged patient. Cortex 38:727-742

Smits DJM, Kuppens P (2005) The relations between anger, coping with anger, and aggression, and the BIS/BAS system. Pers Individ Dif 39:783-793.

Soloff PH, Meltzer CC, Becker C, Greer PJ, Kelly TM, Constantine D (2003) Impulsivity and prefrontal hypometabolism in borderline personality disorder. Psychiatry Res 123:153-163.

Strauss MM, Makrisb N, Aharona I, Vangelb MG, Goodmanb J, Kennedy DN, Gasica GP, Breiter HC (2005) fMRI of sensitization to angry faces. NeuroImage 26:389-413.

Tiihonen J, Kuikka J, Bergstrom K, Hakola P, Karhu J, Ryynanen O-P, Fohr J (1995) Altered striatal dopamine reuptake site densities in habitually violent and non-violent alcoholics. Nat Med 1:654-657.

Tottenham N, Borsheid A, Ellertsen K, Marcus DJ, Nelson CA (2002) Categorization of facial expressions in children and adults: establishing a larger stimulus set. Paper presented at the Cognitive Neuroscience Society Annual Meeting, San Francisco, CA, April.

Urry HL, van Reekum CM, Johnstone T, Kalin NH, Thurow ME, Hillary S, Jackson CA, Frye CJ, Greischar LL, Alexander AL, Davidson RJ (2006) Amygdala and ventromedial prefrontal cortex are inversely coupled during regulation of negative affect and predict the diurnal pattern of cortisol secretion among older adults. J Neurosci 26:4415-4425.

van Honk J, Tuiten A, Hermans E, Putman P, Koppeschaar H, Thijssen J, Verbaten R, van Doornen L (2001) A single administration of testosterone induces cardiac accelerative responses to angry faces in healthy young women. Behav Neurosci 15:238-242.

van Reekum CM, Urry HL, Johnstone T, Thurow ME, Frye CJ, Jackson CA, Schaefer HS, Alexander AL, Davidson RJ (2007) Individual differences in amygdala and ventromedial prefrontal cortex activity are associated with evaluation speed and psychological well-being. J Cogn Neurosci 19:237-248.

Vukhac K-L, Sankoorikal E-B, Wang Y (2001) Dopamine D2L receptorand age-related reduction in offensive aggression. NeuroReport 12:1035-1038.

Whalen PJ, Shin LM, McInerney SC, Fischer H, Wright CI, Rauch SL (2001) A functional MRI study of human amygdala responses to facial expressions of fear vs. anger. Emotion 1:70-83.

Wingrove J, Bond AJ (1998) Angry reactions to failure on a cooperative computer game: the effect of trait hostility, behavioural inhibition, and behavioural activation. Aggress Behav 24:27-36.

Worsley KJ, Marrett S, Neelin P, Vandal AC, Friston KJ, Evans AC (1996) A unified statistical approach for determining significant signals in images of cerebral activation. Hum Brain Mapp 4:58-83. 